RESEARCH ARTICLE

\title{
Pattern of Surgical Antibiotic Prophylaxis for Surgical Site Infections in at two Teaching Hospitals, Islamabad, Pakistan
}

\author{
Zakir Khan ${ }^{1,2}$ Naveed Ahmed ${ }^{1}$, Asim-ur-Rehman ${ }^{1}$, Faiz Ullah Khan ${ }^{3,4}$, Hazir Rahman ${ }^{5}$ \\ ${ }^{1}$ Department of Pharmacy Quaid-i-Azam University Islamabad, Pakistan \\ ${ }^{2}$ Institute of Health Sciences, Department of Pharmacology (Pharmacovigilance), Çukurova University, Adana, \\ Turkey \\ ${ }^{3}$ Department of Pharmacy Administration and Clinical Pharmacy, School of Pharmacy, Health Science Centre, Xi'an \\ Jiaotong University, China \\ ${ }^{4}$ Department of Pharmacy Quaid-i-Azam University Islamabad, Pakistan \\ ${ }^{5}$ Department of Microbiology, Faculty of Chemical and Life Sciences, Abdul Wali Khan University, Mardan, Pakistan
}

\begin{abstract}
Objectives: To investigate the utilization pattern of Surgical antibiotic prophylaxis (SAP) for the prevention of surgical infections in most common abdominal and orthopedic surgical procedures.

Methods: A prospective observational and non-intervention study was conducted at two teaching hospitals (PIMS and $\mathrm{SIH}$ ) in Islamabad, Pakistan. Prescriptions records were reviewed to assess the utilization pattern of SAP during the nine-month duration. Data regarding most common surgical procedures, antimicrobial utilization, dose, route and administration timing of SAP were extracted for analysis. Observed practices were compared with standard treatment guidelines.

Results: Out of total 1512 surgical procedures about, $56.9 \%(n=860)$ of patients performed abdominal followed by $652(43.1 \%)$ orthopedic surgical procedures. A total of $212(14 \%)$ surgical procedures were completely correct in all steps. SAP were given in $1474(97.5 \%)$ of surgical procedures. In 712 of the participants $(48.3 \%)$ were given Ceftriaxone following by Cefazolin $(n=247,16.7 \%)$. Abdominal surgeries were majorly managed with Ceftriaxone $(59 \%)$ while the orthopedic surgeries managed with Ceftriaxone (31.3\%) and Cefazolin (30\%). Appropriate choice of SAP was observed in $(n=275 ; 18.6 \%)$ procedures and about half $(n=719,49 \%)$ received antibiotics within optimal timing. The appropriate choice/selection of SAP according to the guidelines was greater in orthopedic surgical procedures $(n=212 ; 14.8 \%)$ than abdominal surgeries $(n=63 ; 4.3 \%) ; p \leq 0.001$. Compliance with respect to timing was significantly lower in an orthopedic surgical procedure $(n=301)$ as compared to abdominal surgeries $(n=418)$; $p \leq 0.001$. Similarly, a statistically significant difference observed between PIMS and SIH with respect to SAP practices; $p \leq 0.001$.
\end{abstract}

Conclusions: Inappropriate choice, the timing of administration and combination of SAP were observed in the current study. J Microbiol Infect Dis 2019; 9(3):104-111.

Keywords: Surgery, perioperative, antibiotic, prophylaxis, hospital

\section{INTRODUCTION}

Surgical site infection (SSI) is one of the drastic post-operative complications and ultimately affect the overall quality of life due to increased morbidity, mortality and treatment cost $[1,2]$. Surgical antibiotic prophylaxis (SAP) administration before surgery is an evidencebased practice for the prevention of SSI $[3,4]$. However, the nature of the pathogen, pharmacokinetic profile of therapeutic agent, correct timing, dose and route according to the patient and surgical procedure are crucial for appropriate prophylaxis [1]. Despite the authentic evidence about the SAP effectiveness, its use often inappropriately reported by different studies $[1,2,5]$. Inappropriate choice, timing and the duration of the SAP were commonly reported $[1,2,6]$. As a result, irrational use of SAP ultimately leads to the increased cost of therapy, resistance and adverse drug reaction [2-4]. 
The recently published study in Pakistan, demonstrated that the SSI rates were ranged from $6.5-8.3 \%$ which is much higher as compared developed countries [7]. Inappropriate usage of SAP, high prevalence of multi-drug resistance, unavailability of local guidelines and limited available research on the pattern of SAP use in Pakistani hospital are still a problem [7-9]. Previous reports indicate that at least one antimicrobial is prescribed to $30-50 \%$ of all hospitalized patients, which is responsible for more than $30 \%$ of hospital budget [10]. Furthermore, SAP occurs in one-third of all antibiotic use in hospitals and $80 \%$ of all antibiotic use in surgery [11]. However, despite its extensive utilization, the detail usage patterns of antimicrobials as SAP for SSIs prevention are poorly explored in selected surgical procedures. Furthermore, no similar investigations were carried out in selected tertiary care teaching hospitals. The aim of this prospective crosssectional study was to investigate the utilization pattern of antimicrobial as SAP in most common abdominal and orthopedic surgical procedures.

\section{METHODS}

This prospective cross-sectional study was conducted in six most common surgical procedures (acute appendectomy, laparoscopic cholecystectomy, inguinal hernia, total knee replacement, total hip replacement and debridement surgery procedures) at Pakistan Institute of Medical Sciences hospital (A government based hospital; PIMS) and Shifa International hospital (A private based hospitals; $\mathrm{SIH})$ from August 18, 2016 to May 18, 2017 at the Capital city (Islamabad) of Pakistan. These hospitals are 600-bed, teaching and main referral hospitals in the capital city, Islamabad, Pakistan. They provide medical, surgical and emergency care to the population of Islamabad, Khyber Pakhtunkhwa, Punjab and Azad Jammu Kashmir. The study was approved by the University Bio-ethics committee and Institutional review boards of concerned hospitals.

A prospective, observational, prescription based and the non-interventional study was carried out for appropriate utilization and pattern of SAP in most common abdominal and orthopedic surgical procedures. Medical records with missing information, less commonly performed surgical procedures, and those patients who performed more than one surgical procedure were excluded from the study analysis. The modified pretested data collection form was used for the extraction of important information from medical records. Details of medical records related to surgery type, antimicrobial choice, route of administration, the timing of administration, dosage form and dose were collected. Furthermore, data were crosschecked by a principal investigator, collaborators in surgical ward and resident pharmacist for correct information.

The observed practices were compared with standard treatment guidelines of 'American College of Surgeons and Surgical Infection Society: Surgical Site Infection Guidelines' [3] and 'American Society of Health-System Pharmacists' [4]. These guidelines emphasized on the following aspects; a) use of inexpensive narrow-spectrum antibiotics b) intravenous single dose prophylaxis $\mathrm{c}$ ) administration of SAP within 1 hour before the first incision d) $A$ Cefazolin is the first drug of choice, however, if there is allergy to beta-lactams then Vancomycin or Clindamycin should be appropriate alternative regime (Metronidazole should be added against anaerobic microorganism activity) f) dose of SAP. The investigation of appropriate SAP practices was based on 1) appropriate choice of SAP according to surgical procedures 2) route 3) dose 4) timing of administration. Furthermore, the utilization pattern of antibiotics was also determined by the World Health Organization/ Anatomical Therapeutic Classification System [12]. Finally, the collected data was entered to SPSS version 22.0 for descriptive (frequency and percentages) and Chi-square test analysis.

\section{RESULTS}

A total of 1512 eligible surgeries were observed according to the inclusion criteria of study from both hospitals. Out of these 860 patients underwent abdominal surgical procedures ( $n=450$ in PIMS and $n=410$ in SIH) while 652 patients with orthopedic surgeries $(n=322$ in PIMS and $n=330$ in SIH). An acute appendectomy $(n=398,46.3 \%)$ were the most common abdominal $(n=289,44.3 \%)$ surgeries and total knee replacement were the most common orthopedic surgical procedures. Details about surgical procedures performed are shown in Table 1. 


\section{Evaluation of SAP Practices}

\section{SAP indication}

Prophylactic antibiotics were given in 1,474 $(97.5 \%)$ of surgical procedures and about $2.5 \%$ $(n=38)$ patients did not receive SAP (Table 1).

\section{SAP Choice}

With respect to commonly prescribed antimicrobials, about half of the participants 712 (48.3\%) were given Ceftriaxone following by cefazolin $(\mathrm{n}=230,15.6 \%)$ and cefuroxime $(n=128, \quad 8.7 \%)$. The combination of cefoperazone plus sulbactam $(n=101,6.8 \%)$ and amoxicillin plus Clavulanic acid ( $n=94,6.4 \%$ ) were also prescribed as SAP. However, a drug of choice Cefazolin was only prescribed in 247 (16.8\%), Vancomycin $3 \quad(0.2 \%)$ and Metronidazole $25(1.7 \%)$ procedure as SAP (Table 2). The use of appropriate SAP choice was greater in Orthopedic surgical procedures $(n=212 ; 14.8 \%)$ than abdominal surgeries $(n=63$; 4.3\%); $P=0.001$. A statistically significant difference was also observed between the hospital setting and correct antibiotic choice $(\mathrm{P}<0.05)$ (Table 2).

\section{Dose of SAP}

Concerning the dosage, a single dose of SAP was prescribed in both abdominal and orthopedic surgeries. A $2 \mathrm{~g}$ dose was used for both ceftriaxone and cefazolin, $1 \mathrm{~g}$ for Cefoperazone plus Sulbactam and $1.2 \mathrm{~g}$ for amoxicillin Plus Clavulanic acid as SAP (Table 2).

\section{SAP Route and timing of administration}

All patients received SAP through intravenous route and about, $48.8 \% \quad(n=719)$ patients received SAP according to the recommended timing of administration. Compliance with respect to timing was significantly lower in an orthopedic surgical procedure $(n=301)$ as compared to abdominal surgeries $(n=418)$; $\mathrm{P}=0.001$ (Table 1). Similarly, a statistically significant difference observed between PIMS and $\mathrm{SIH} ; \mathrm{P}=0.01$ (Table 2).

\section{Total appropriateness assessment}

A total of 212 (14\%; PIMS: $n=98, S I H: n=114)$ surgical procedures were completely correct in all steps (Table 2).

\section{Utilization pattern of SAP according to procedures}

\section{Abdominal surgical procedures}

Related to selection of SAP for abdominal procedures, about $77.6 \%$ (82.4\% in PIMS and $69.6 \%$ in $\mathrm{SIH}$ ) of patients managed with ceftriaxone in an acute appendectomy, 55.8\% $(56.6 \%$ in PIMS and $55.4 \%$ in $\mathrm{SIH}$ ) in laparoscopic cholecystectomy, while half of the inguinal surgeries patients $(51.2 \%)$ received amoxicillin/Clavulanic acid (56.3\% in PIMS and $46.4 \%$ in $\mathrm{SIH}$ ). The remaining patients were managed with other different types of regimens. In the current study, out of 835 patients, whose received SAP in abdominal surgeries, the timing of administration was appropriate (within 1 hour before surgery) in half $(51 \%)$ of the procedures (Table 1 and 2).

\section{Orthopedic surgical procedures}

With respect to orthopedic surgical procedures, the cefazolin was prescribed to $35.6 \%$ of total knee replacement and $39 \%$ of total hip replacement surgical patients. However, debridement surgery was mainly managed through ceftriaxone (49.7\%). About, $47 \%$ of orthopedic surgical patients received SAP within optimal timing (Table 1 and 2).

\section{DISCUSSION}

This study was carried out to investigate the utilization pattern of antibiotic as SAP for SSIs prevention in most common abdominal and orthopedic surgical procedures at two teaching referral hospitals. Most of the patients received SAP who underwent selected common abdominal and orthopedic surgical procedures. The total appropriateness of SAP was low in our study (14\%). These finding was supported by Turkish study (19.7\%) [5], but lower as compared to study conducted in France (41.7\%) [13]. The inappropriate use of SAP may be due to unavailability of guidelines on SAP in Pakistan $[7,8]$.

The SAP was administered in line with standard guidelines recommendations in $18.6 \% \quad(n=275)$ of patients. The low adherence rate was reported by studies conducted in Italy $(5.7 \%)$ [14] and Brazil (5.8\%) [15]. However, higher adherence rate with respect to an appropriate choice of SAP was reported in Germany (70.7\%) 
[16], Turkey (68\%) [17] Qatar (68\%) [18] and India (52\%) [19]. The first step for the appropriate use of SAP is to provide educational training on antibiotic stewardship program and associated SSIs risk. Compliance with antibiotic prophylaxis guidelines improved with increased awareness among surgeons and other health care team members [20, 21].

Ceftriaxone and cefazolin were the most commonly prescribed agent as SAP, while ceftriaxone alone being given to $48.3 \%$ patients in all surgeries. These findings are aligned with studies conducted in different countries [1,2,6, 22] which indicates over usage of antimicrobials in surgical procedures. This may be due to the lack of updated information, continuous training, awareness, and adherence with international and local evidence-based treatment guidelines among prescribers of surgical wards $[1,2,18]$.

Evidence-based international treatment guideline emphasized on the use of costeffective, narrow strength antimicrobial as SAP $[3,4]$. Cefazolin is the most appropriate and indicated a choice for most of the surgical procedures. However, our study reported that the most common class of antibiotics was Cephalosporins specifically, ceftriaxone was mostly prescribed to managed surgical procedures. These findings are aligned with Ethiopian studies [1, 2] and similar results also reported in Malaysian [23], Turkish [5] and Iranian [22] studies. Whereas, these findings have deviated from previously conducted studies in Singapore, Greece, Germany, and Italy which showed most common antibiotics were cefazolin, ceforanide, cefuroxime, and levofloxacin respectively $[16,18,24,25]$. It is evident that the emergence of extendedspectrum beta-lactamase (ESBL) producing microorganisms arises due to the overuse of third-generation cephalosporins [26]. Unavailability of antimicrobials and nonadherence with guidelines may be a reason behind such practices.

In this study, a combination (cefoperazone plus sulbactam and amoxicillin plus Clavulanic acid) were also prescribed as SAP (13.2\%). Combination therapy also reported by other studies [1, 2, 23], although it is evident that combination therapy creates resistance and socioeconomic problems for patients. Combination therapy only recommended for dirty contaminated procedures or when anaerobic bacterial coverage required $[3,4]$.

Different types of SAP were prescribed for the management of surgical procedures in our study. Such as, appendectomy was managed through ceftriaxone, cefoperazone plus sulbactam and combination of ceftriaxone and metronidazole. Such type of practices also observed in laparoscopic cholecystectomy, inguinal hernia, total knee replacement surgeries, and others. These practices are deviated from recommendations of guidelines and also reported in other studies $[1,2,22,23]$.

Table 1. Surgical antimicrobial prophylaxis in selected surgical procedure $(n=1512)$

\begin{tabular}{lccccc}
\hline Indicators & $\begin{array}{c}\text { Antibiotic } \\
\text { given }\end{array}$ & $\begin{array}{c}\text { Non-use of } \\
\text { antibiotic }\end{array}$ & $\begin{array}{c}\text { Most common } \\
\text { antibiotic with dose }\end{array}$ & Route & \multicolumn{2}{c}{$\begin{array}{c}\text { Timing } \\
\text { On time }\end{array}$} \\
Late
\end{tabular}


Table 2. Frequency and percentages of various SAP prescribed in selected surgical procedures in Pakistan Institute of Medical Sciences ( $\mathrm{n}=772$ ).

\begin{tabular}{|c|c|c|c|c|c|c|}
\hline Antimicrobials & Appendectomy & $\begin{array}{c}\text { Laparoscopic } \\
\text { cholecystectomy }\end{array}$ & $\begin{array}{l}\text { Inguinal } \\
\text { hernia }\end{array}$ & $\begin{array}{l}\text { Total Knee } \\
\text { replacement }\end{array}$ & Hip replacement & $\begin{array}{l}\text { Debridement } \\
\text { surgery }\end{array}$ \\
\hline CRO (2 g) & $206(82.4)$ & $64(56.6)$ & $18(20.7)$ & $49(31.6)$ & $14(14.2)$ & $6(8.7)$ \\
\hline CFZ (2 g) & $2(0.8)$ & 7 (6.2) & $11(12.6)$ & $53(34.1)$ & $43(43.9)$ & $23(33.3)$ \\
\hline CXM $(1.5 \mathrm{~g})$ & $0(0)$ & $20(17.7)$ & $6(6.9)$ & $0(0)$ & $29(29.6)$ & $16(23.1)$ \\
\hline CIP $(500 \mathrm{mg})$ & $6(2.4)$ & $0(0)$ & $0(0)$ & $0(0)$ & $0(0)$ & $0(0)$ \\
\hline CRO (2 g) + MTZ (500 mg) & $7(2.8)$ & $0(0)$ & $0(0)$ & $0(0)$ & $0(0)$ & $0(0)$ \\
\hline $\mathrm{C} / \mathrm{S}(1 \mathrm{~g})$ & $18(7.2)$ & $0(0)$ & $0(0)$ & $22(14.1)$ & $0(0)$ & $0(0)$ \\
\hline AMC (1.2 g) & $6(2.4)$ & $3(2.7)$ & $49(56.3)$ & $0(0)$ & $0(0)$ & $0(0)$ \\
\hline TZP $(4.5 \mathrm{~g})$ & $4(1.6)$ & $0(0)$ & $0(0)$ & $0(0)$ & $0(0)$ & $9(13)$ \\
\hline VAN (500 mg) & $1(0.4)$ & $0(0)$ & $0(0)$ & $0(0)$ & $0(0)$ & $0(0)$ \\
\hline AZM (500 mg) & $0(0)$ & $13(11.5)$ & $0(0)$ & $0(0)$ & $0(0)$ & $0(0)$ \\
\hline AMK (500 mg) & $0(0)$ & $2(1.8)$ & $0(0)$ & $10(6.4)$ & $0(0)$ & $0(0)$ \\
\hline RAD (500 mg) & $0(0)$ & $0(0)$ & $0(0)$ & $0(0)$ & $0(0)$ & $11(15.9)$ \\
\hline FEP $(2 \mathrm{~g})$ & $0(0)$ & $0(0)$ & $0(0)$ & $12(7.7)$ & $8(8.1)$ & $0(0)$ \\
\hline CXM or CFZ $(2 \mathrm{~g}+2 \mathrm{~g})$ & $0(0)$ & $0(0)$ & $0(0)$ & $9(5.8)$ & $0(0)$ & $0(0)$ \\
\hline Non-use of SAP & $0(0)$ & $4(3.5$ & $3(3.4)$ & $0(0)$ & $4(4.5)$ & $4(5.8)$ \\
\hline Correct Choice & $10(4)$ & 7 (6.2) & $11(12.6)$ & $53(34.1)$ & $43(43.9)$ & $23(33.3)$ \\
\hline Correct Timing & $89(35.6)$ & $43(39.5)$ & $31(36.9)$ & $81(52.2)$ & $22(23.4)$ & $11(16.9)$ \\
\hline Inappropriate timing & $161(64.4)$ & $66(60.5)$ & $53(63.1)$ & $74(47.8)$ & $72(76.6)$ & $54(83.1)$ \\
\hline Total appropriateness $(\mathrm{n}=98)$ & $7(2.8)$ & $2(1.8)$ & $9(10.3)$ & $34(21.9)$ & $29(29.6)$ & $17(24.6)$ \\
\hline Total surgeries & $250(100)$ & $113(100)$ & $87(100)$ & $155(100)$ & $98(100)$ & $69(100)$ \\
\hline
\end{tabular}


Table 3. Frequency and percentages of various SAP prescribed in selected surgical procedures in Shifa International Hospital ( $\mathrm{n}=740$ ).

\begin{tabular}{|c|c|c|c|c|c|c|}
\hline Antimicrobials & Appendectom! & $\begin{array}{c}\text { Laparoscopic } \\
\text { cholecystectomy }\end{array}$ & $\begin{array}{l}\text { Inguinal } \\
\text { hernia }\end{array}$ & $\begin{array}{c}\text { Total Knee } \\
\text { replacement }\end{array}$ & $\begin{array}{c}\text { Hip } \\
\text { replacement }\end{array}$ & $\begin{array}{c}\text { Debridement } \\
\text { surgery }\end{array}$ \\
\hline CRO $(2 \mathrm{~g})$ & $103(69.6)$ & $107(55.4)$ & $10(14.49)$ & $43(32)$ & $22(19.6)$ & $53(63)$ \\
\hline CFZ (2 g) & $0(0)$ & $11(5.7)$ & $4(5.8)$ & 50 (37.3) & $39(34.8)$ & $4(4.7)$ \\
\hline CXM $(1.5 \mathrm{~g})$ & $0(0)$ & $0(0)$ & $14(20.3)$ & $0(0)$ & $33(29.4)$ & $10(11.9)$ \\
\hline $\begin{array}{l}\text { CRO }(2 \mathrm{~g})+\mathrm{MTZ}(500 \\
\mathrm{mg})\end{array}$ & $18(12.16)$ & $0(0)$ & $0(0)$ & $0(0)$ & $0(0)$ & $0(0)$ \\
\hline $\mathrm{C} / \mathrm{S}(1 \mathrm{~g})$ & $25(16.9)$ & $0(0)$ & $0(0)$ & $36(26.8)$ & $0(0)$ & $0(0)$ \\
\hline $\operatorname{AMC}(1.2 \mathrm{~g})$ & $2(1.2)$ & $2(1)$ & $32(46.4)$ & $0(0)$ & $0(0)$ & $0(0)$ \\
\hline TZP (4.5 g) & $0(0)$ & $18(9.3)$ & $0(0)$ & $0(0)$ & $0(0)$ & $0(0)$ \\
\hline VAN (500 mg) & $0(0)$ & $2(1)$ & $0(0)$ & $0(0)$ & $0(0)$ & $0(0)$ \\
\hline AZM (500 mg) & $0(0)$ & $35(18.1)$ & $0(0)$ & $0(0)$ & $0(0)$ & $3(3.6)$ \\
\hline AMK (500 mg) & $0(0)$ & $1(0.5)$ & $6(8.7)$ & $0(0)$ & $0(0)$ & $0(0)$ \\
\hline RAD (500 mg) & $0(0)$ & $0(0)$ & $2(2.9)$ & $0(0)$ & $0(0)$ & $8(9.5)$ \\
\hline $\operatorname{FEP}(2 \mathrm{~g})$ & $0(0)$ & $0(0)$ & $0(0)$ & $5(3.7)$ & $17(15.1)$ & $2(2.4)$ \\
\hline Non-use of SAP & $0(0)$ & $17(8.8)$ & $1(1.4)$ & $0(0)$ & $1(0.9)$ & $4(4.7)$ \\
\hline Correct Choice & $18(12.16)$ & $13(6.7)$ & $4(5.8)$ & $50(37.3)$ & $39(34.8)$ & $4(4.7)$ \\
\hline Correct Timing & $94(63.5)$ & $114(64.8)$ & $47(69.1)$ & $91(67.9)$ & $63(56.8)$ & $33(41.2)$ \\
\hline Inappropriate Timing & $54(36.5)$ & $62(35.2)$ & $21(30.9)$ & $43(32.1)$ & 48 (43.2) & 47 (58.8) \\
\hline Total Appropriate $(n=114)$ & $15(10.1)$ & $12(6.2)$ & $3(4.3)$ & $47(35)$ & $35(31.2)$ & $2(2.3)$ \\
\hline Total surgeries & $148(100)$ & $193(100)$ & $69(100)$ & $134(100)$ & $112(100)$ & $84(100)$ \\
\hline
\end{tabular}

IV Intravenous, $\mathrm{n}=$ Number, $\mathrm{CRO}=$ Ceftriaxone, $\mathrm{AMC}=\mathrm{Amoxicillin+Clavulaunic}$ acid, $\mathrm{CFZ}=$ Cefazolin, $\mathrm{CXM}=$ Cefuroxime, $\mathrm{C} / \mathrm{S}=\mathrm{Cefoperazone} / \mathrm{Sulbactam}, \mathrm{AZM}=\mathrm{Azithromycin}, \mathrm{AMK}=$ Amikacin, $R A D=$ Cephradine $F E P=C e f e p i m, C X M=$ Cefuroxime, $S A P=S u r g i c a l$ antimicrobial prophylaxis, $S I=S u r g i c a l$ incision 
Regarding SAP timing of administration, in our study about half $(\mathrm{n}=719,49 \%)$ received antibiotics within 30-60 minutes before surgical incision which, indicating half percent of adherence to recommendations of guidelines. A similar finding was reported by an Australian study [21]. Low adherence rate was reported in Nigeria [20] and Egypt [27] with respect to the appropriate timing of administration. However, the higher adherence rate was reported in Jorden (99\%) [22], England (86.4\%) [28] and Palestine $(76.6 \%)$ [29]. It is the best evidence that delayed administration of SAP is associated with 2-time greater risk of SSIs as compared to timely administration [20]. Appropriate time of administration of PPA can also reduce the duration of stay and hospitalization cost [3, 4]. Non-availability of standard guidelines and protocols for antibiotic prophylaxis in these hospitals could be a reason for non-compliance in our study [2,20].

The current study had access to well-reported data on SAPs and was adequately powered, some limitations must be acknowledged. First, Present study only focused on the compliance rate of SAPs in only common surgical procedures in our settings. Second, we used published recommendations of evidence-based international guidelines to measure against the appropriate use of SAPs in selected surgeries. Because there were no local consensus guidelines available in both hospitals. However, the possibility exists that recommendations given by the guidelines were not practicable in our patients or for the situation in Pakistan. We conducted a prescription based observational and non-interventional study. Therefore, we did not know if the overuse of broad-spectrum antibiotics had any clinical consequences. Finally, these findings do however add a piece of useful information, particularly around appropriate SAP use, timing of administration and adherence with standard guidelines in surgical procedures and health systems in developing countries.

In conclusion, this study reported that either as a single or combined form of antimicrobial as SAP received by most of the patients. Inappropriate choice, the timing of administration and the combination of antimicrobial for the prevention of surgical infections were observed. Continuous educational training, availability of appropriate antimicrobial, implementation of local and international treatment guidelines and antimicrobial stewardship program are required for appropriate utilization of SAP.

\section{ACKNOWLEDGMENTS}

The authors acknowledge the administration of both hospitals for their supports and help during data collection.

Declaration of Conflicting Interests: The authors declare that they have no conflict of interest.

Funding: This project received no specific grant from any funding agency in the public, commercial, or not-for-profit sectors.

\section{REFERENCES}

1. Alemkere G. Antibiotic usage in surgical prophylaxis: A prospective observational study in the surgical ward of Nekemte Referral Hospital. PloS one 2018; 13(9):e0203523.

2. Ayele $\mathrm{Y}$, Taye $\mathrm{H}$. Antibiotic utilization pattern for surgical site infection prophylaxis at Dil Chora Referral Hospital Surgical Ward, Dire Dawa, Eastern Ethiopia. BMC Res Notes 2018; 11(1):537.

3. Ban KA, Minei JP, Laronga C, et al. American College of Surgeons and Surgical Infection Society: Surgical Site Infection Guidelines, 2016 Update. J Am College of Surgeons 2017; 224(1):59-74.

4. Bratzler DW, Dellinger EP, Olsen KM, et al. Clinical practice guidelines for antimicrobial prophylaxis in surgery. Surgical infections, 2013. 14(1): p. 73-156.

5. Hosoglu S, Aslan S, Akalin S, Bosnak V. Audit of quality of perioperative antimicrobial prophylaxis. Pharmacy World \& Science 2009: 31(1):14-17.

6. Nabovati E, Vakili-Arki H, Taherzadeh Z, Hasibian MR, Abu-Hanna A, Eslami S. Drug-drug interactions in inpatient and outpatient settings in Iran: a systematic review of the literature. DARU J Pharmaceutic Sci 2014: 22(1):52.

7. Butt SZ, Ahmad M, Saeed Hamid, Saleem Z, Javaid Z. Post-surgical antibiotic prophylaxis: Impact of pharmacist's educational intervention on appropriate use of antibiotics. J Infect Public Health 2019. https://doi.org/10.1016/j.jiph.2019.05.015.

8. Khan Z, Ahmed N, Rehman AU, Khan FU, Rahman $H$. Utilization of Antibiotic Prophylaxis in Three Common Abdominal Surgeries, Adherence to Standard Guidelines and Surgeons' Perception in 
Teaching Hospitals, Islamabad, Pakistan. MDPI preprint 2019: 2019060119. https://www.preprints.org/manuscript/201906.0119/v1.

9. Nausheen S, Hammad R, Khan A. Rational use of antibiotics--a quality improvement initiative in hospital setting. J Pak Med Assoc 2013; 63(1):60.

10. Atif M, Azeem M, Sarwar MR, et al. WHO/INRUD prescribing indicators and prescribing trends of antibiotics in the accident and emergency department of Bahawal Victoria hospital, Pakistan. Springerplus 2016; 5(1):1928.

11. Hansen S, Sohr D, Piening B, et al. Antibiotic usage in German hospitals: results of the second national prevalence study. J Antimicrob Chemother 2013; 68(12):2934-2939.

12. World Health organization ATC/DDD Methodology. 2018. World Health organization (WHO) ATC/DDD Methodology. 2018. Available from< https://www.who.int/medicines/regulation/medicinessafety/toolkit_methodology/en/>. Accessed 12 December 2018.

13. Martin C, Pourriat J. Quality of perioperative antibiotic administration by French anaesthetists. J Hosp Infect 1998; 40(1):47-53.

14. Giordano M, Squillace L, Pavia M. Appropriateness of surgical antibiotic prophylaxis in pediatric patients in Italy. Infect Control Hosp Epidemiol 2017; 38(7):823-831.

15. Schmitt C, Lacerda RA, Padoveze MC, Turrini RN. Applying validated quality indicators to surgical antibiotic prophylaxis in a Brazilian hospital: learning what should be learned. Am J Infect Control 2012; 40(10):960-962.

16. Hohmann C, Eickhoff C, Radziwill R, Schulz M. Adherence to guidelines for antibiotic prophylaxis in surgery patients in German hospitals: a multicentre evaluation involving pharmacy interns. Infection 2012; 40(2):131-137.

17. Hosoglu S, Sunbul M, Erol S, et al, A national survey of surgical antibiotic prophylaxis in Turkey. Infect Control Hosp Epidemiol 2003; 24(10):758-761.

18. Tourmousoglou CE, Yiannakopoulou ECh, Kalapothaki V, Bramis J, Papadopoulos J. Adherence to guidelines for antibiotic prophylaxis in general surgery: a critical appraisal. J Antimicrob Chemother 2007; 61(1):214-218.

19. Parulekar L, Soman R, Singhal T, Rodrigues C, Dastur FD, Mehta A. How good is compliance with surgical antibiotic prophylaxis guidelines in a tertiary care private hospital in India? A prospective study. Indian J Surg 2009; 71(1):15-18.

20. Abubakar U, Sulaiman SS, Adesiyun A. Utilization of surgical antibiotic prophylaxis for obstetrics and gynaecology surgeries in Northern Nigeria. Int $\mathrm{J}$ Clin Pharm 2018; 40(5):1037-1043.
21. Jaber S, Rogers C, Sunderland B, et al. Appropriateness of surgical antibiotic prophylaxis for breast surgery procedures. Int J Clin Pharm 2017; 39(2):483-486.

22. Al-Momany NH, Al-Bakri AG, Makahleh ZM, Wazaify MM. Adherence to international antimicrobial prophylaxis guidelines in cardiac surgery: a Jordanian study demonstrates need for quality improvement. J Manag Care Pharm 2009; 15(3):262-271.

23. Oh AL, Goh LM, Nik Azim NA, Tee CS, Shehab PCW. Antibiotic usage in surgical prophylaxis: a prospective surveillance of surgical wards at a tertiary hospital in Malaysia. J Infect Dev Ctries 2014; 8(02):193-201.

24. Young B, Ng TM, Teng C, Ang B, Tai HY, Lye DC. Non-concordance with Surgical Site Infection Prevention Guidelines and the Rate of Surgical Site Infections for General Surgical, Neurological and Orthopedic Procedures. Antimicrob Agents Chemother 2011; 55(10):4659-63.

25. Napolitano F, Izzo MT, Giuseppe G, Angelillo IF. Evaluation of the appropriate perioperative antibiotic prophylaxis in Italy. PloS one 2013; 8(11):e79532.

26. Shiva F, Ghanaie R, Shirvani FShiva, et al. Pattern of Antibiotic Usage in Children Hospitalized for Common Infectious Diseases. Arch Pediatr Infect Dis 2018. 2018; 6(1):e34474.

27. Saied T, Hafez SF, Kandeel A, et al. Antimicrobial stewardship to optimize the use of antimicrobials for surgical prophylaxis in Egypt: A multicenter pilot intervention study. Am J Infect Control 2015; 43(11):e67-e71.

28. Hawn MT, Itani KM, Gray SH, Vick CC, Henderson W, Houston TK. Association of timely administration of prophylactic antibiotics for major surgical procedures and surgical site infection. J Am Coll Surg 2008; 206(5):814-819.

29. Musmar SM, Baba H. Adherence to guidelines of antibiotic prophylactic use in surgery: a prospective cohort study in North West Bank, Palestine. BMC Surg 2014; 14(1):69. 